\title{
Emotional and disability status in patients with chronic low back pain
}

\begin{abstract}
Objective: to analyze the prevalence of psychological disorders (anxiety, depression, stress) and the relationship of these factors with functional disability in patients with chronic low back pain. Study design: cross-sectional descriptive and observational study.

Setting: the physiotherapy outpatient clinic of the Northen University of Paraná, Londrina, Paraná, Brazil.

Methods: 84 individuals were recruited, being 43 elderly ( 20 with low back pain and 23 control subjects) and 41 adults ( 21 with low back pain and 20 control subjects). In order to assess the psychological aspects, Beck Inventories of Depression and anxiety as well as Lipp Stress Inventory were used. To evaluate the funcionality, Roland Morris Questionnaire was chosen. The evaluation of the pressure pain threshold was performed using algometer emg systems ${ }^{\circledR}$. To analyse was considered a significance level of $5 \%(p<0.05)$.

Results: It was observed that people with low back pain have higher scores of depression, anxiety, stress and functional disability compared to the control group $(p<0.05)$. Among adults, an inverse relationship between anxiety and disability $(p<0.05)$ was observed. In subjects with low back pain positive correlation was observed between anxiety and functional disability and, between the depression and functional disability $(p<0.05)$. In addition, no association was found between stress and functional disability in patients with low back pain $(p<0.05)$. Conclusion: suggest that in patients with low back pain worsening of functionality is anxiety and stress and elderly people with low back pain has higher levels of stress, depression, anxiety and function disability.
\end{abstract}

Keywords: low back pain, anxiety, depression, aged, physical functional performance, disability evaluation
Volume 7 Issue 2 - 2020

\author{
Mayra Campos Frâncica dos Santos,' João \\ Paulo Manfre dos Santos, ${ }^{2}$ Rubens Alexandre \\ Silva Júnior, ${ }^{3}$ Rosângela Aparecida Pimenta \\ Ferrari, ${ }^{4}$ Ligia Megumi lida,' Rodrigo Antônio \\ Carvalho Andraus, ${ }^{3}$ Sue Ellen Ferreira \\ Modesto Ruy Figueiredo,' Karen Barros \\ Parron Fernandes ${ }^{3,5}$ \\ 'Master Program in Rehabilitation Sciences (UEL/UNOPAR), \\ Brazil \\ ${ }^{2}$ Doctoral Program in Rehabilitation Sciences (UEL/UNOPAR), \\ Brazil \\ ${ }^{3}$ Associate Professor, Doctoral Program in Rehabilitation \\ Sciences (UEL/UNOPAR), Brazil \\ ${ }^{4}$ Post doctor of the Program in Rehabilitation Sciences, \\ University of Northern Paraná (UNOPAR), Brazil \\ ${ }^{5}$ Manager of Education and Research, Institute of Education, \\ Research and Innovation, Irmandade da Santa Casa de Londrina \\ (IEPI-ISCAL), Brazil
}

Correspondence: Karen Barros Parron Fernandes, Biological and Health Sciences Center-UNOPAR, Marselha, 59I-Jd. Piza, CEP: 8604 I- I 40-Londrina, PR, Brasil, Tel +55(43)337I7990, Email karenparron@gmail.com

Received: October 22, 2019 | Published: April 30, 2020

\section{Introduction}

The prevalence of chronic non-specific low back pain among the seven countries in the region of the Latin American population was estimated to be around $10.5 \%$. Some risk factors reported by the authors are long working hours with the worker in the sitting position, obesity and overweight, pregnancy, smoking, advanced age, lifting and carrying heavy loads, domestic work, sedentary lifestyles, and duration of current employment. A subgroup analysis of the population under study yielded an estimated prevalence of low back pain of $16.7 \%$ for the population exposed to a lower number of risk factors and $65 \%$ for the higher risk subgroup. ${ }^{1}$ Researchers who estimated worldwide prevalence of chronic low back pain according to age and sex between 1989 and 2013 identified that chronic low back pain prevalence was $4.2 \%$ in individuals aged between 24 and 39 years old and $19.6 \%$ in those aged between 20 and 59. Of nine studies with individuals aged 18 and above, six reported chronic low back pain between $3.9 \%$ and $10.2 \%$ and three, prevalence between $13.1 \%$ and $20.3 \%$. In the Brazilian older population, chronic low back pain prevalence was $25.4 \% .{ }^{2}$ Systematic review of structural and functional brain abnormalities in chronic low back pain (DLC) using MRI identified moderate evidence of regional changes in gray and white matter along with altered functional connectivity during rest and increased activity in areas related to pain after stimulation, evidencing an increased pain matrix. ${ }^{3}$ Therefore, pain should be considered in CLBP, since studies indicate high scores of altered emotional state such as anxiety, depression and somatization in both young adults and the elderly. ${ }^{4,5}$ Despite there are many published guidelines for the diagnosis and treatment of chronic low back pain, few emphasize the psychological issues in the etiology and mainly functional impairment of individuals affected by this condition. Moreover, it is not clear if these psychological problems may still be noticed in adults with CLBP.

Therefore, this study aimed to analyze the prevalence of psychological disorders (anxiety, depression, stress) and the relationship of these factors with functional disability in patients with chronic low back pain.

\section{Materials and methods}

\section{Study design and ethical procedures}

Cross-sectional descriptive and observational study carried out in 2015 following STROBE recommendations for cross-sectional studies. ${ }^{6}$ Research approved by the University's Ethics Committee (Protocol $n^{\circ}$ 956.650) and all participants agreed and signed the free and informed consent form.

\section{Population study}

Participants were recruited through physician contact and previous records in public health services in the city. After inclusion in the 
study, participants were separated into two distinct groups: healthy subjects (G1) and individuals with chronic low back pain (G2).

\section{Eligibility criteria}

1. Inclusion criteria for the healthy group were: absence of reporting low back pain; absence of involvement in physical activity programs; be physically active; accept voluntarily to participate in the study.

2. Inclusion criteria for chronic low back pain group: presence of low back pain or lumbosacral with or without irradiation limited to the knees, through self-report and confirmed by physician; absence of involvement in rehabilitation programs.

3. Exclusion criteria for both groups: presence of any neurological orthopedic, vestibular or vascular diseases.

\section{Intervention}

\section{Low back pain individuals' profile}

In order to characterize the profile of individuals with chronic low back pain, a structured questionnaire was applied. Moreover, cognitive status using the Mini Mental Exam with a cut-off point regarding scholar background ${ }^{7}$ was assessed to apply the exclusion criterion.

\section{Evaluation of emotional aspects}

One psychologist used the Beck Inventory of Depression and Anxiety (BDI) as well as the Inventory of Stress Symptoms for all participants. It is the measure of self-assessment of depression widely used both in research and practice. ${ }^{8,9}$ It is a self-report scale with 21 items, each with four options, with increasing degrees of severity of depression which include symptoms and attitudes suitable for subjects 17 to 80 years old. ${ }^{10}$ The total score is the sum of the individual scores of the items and allows the classification of severity of depression levels. Considering the recommendations of the "Center for Cognitive Therapy", the following cut-off points are recommended: less than $10=$ no or minimal depression; $10-18=$ depression, mild to moderate; $19-29=$ depression, moderate to severe; $30-63=$ severe depression ${ }^{9}$

\section{Beck anxiety inventory (BAI)}

This questionnaire was created to evaluate different levels of anxiety disorders. ${ }^{11}$ It consists of a series of 21 multiple choice questions with four possible answers, with self-assessment questions on the perception of anxiety for the patient throughout the week that has passed. The questions assess the various symptoms of anxiety such as sweating, palpitations, irritability, and lack of air. ${ }^{9}$ The sum of individual scores is the total score, which can range between 0 and 63, the classification of the BAI should follow the score: $0-7$ points: indicates minimum level of anxiety; $8-15$ points: mild anxiety; $16-25$ points: moderate anxiety; 26-63 points: severe anxiety. ${ }^{10}$

\section{Inventory of stress symptoms for adults (ISSL)}

It is a useful test to identify characteristic frames of stress, allowing diagnose if the adult has or no stress and in what stage of stress the individual may be classified. Therefore, it points the area of greatest vulnerability, since it allows to identify what kind of symptoms are the most evident (whether physical or somatic symptoms). ${ }^{11}$

\section{Evaluation of pain pressure threshold}

The evaluation of the pressure pain threshold was performed using algometer EMG Systems ${ }^{\circledR}$. The equipment consists of a flat circular tip $1 \mathrm{~cm}^{2}$ by means of which a steadily increasing pressure was applied perpendicularly to the anatomical landmarks pressure. It has sensors connected to a load cell and amplifiers, so the response is sent to the software that provides, through its interface, the pain scale and pressure range. After signal recording, the parameters were extracted through routine in Matlab Program ${ }^{\circledR}$. Prior to the evaluation, participants were familiar with the equipment and the experimental protocol and they were placed in the prone position on a stretcher. A constant and growing pressure of $1 \mathrm{kgf} / \mathrm{cm}^{2} / \mathrm{s}$ (with $10 \mathrm{kgf}$ limit) was applied and individual pain intensity was assessed. Point 1 of the pain intensity scale was considered the point to assess the pressure pain threshold. In all individuals, an evaluation in different points bilaterally of paraspinal muscle (L1, L3 and L5) was considered, with 30 second intervals between them. As the protocol, an increasing pressure by a trained evaluator and blinded about the condition of the individual $\left(1 \mathrm{kgf} / \mathrm{cm}^{2} / \mathrm{s}\right.$, during 10 seconds) in a controlled temperature room $\left(22^{\circ} \mathrm{C}\right)$ was performed. During each evaluation, the subject was instructed to completely relax, give a full expiration and suspending the breath. The contact surface of the device interface with the skin was $90^{\circ} .{ }^{12}$

\section{Functional assessment}

Roland Morris Disability Questionnaire was used to assess the functional status of individuals with LBP. ${ }^{13}$ It was developed to assess disability in the form of self-reported for patients with low back pain. At this questionnaire, 24 questions involving activities of daily living, pain and functional status with scores ranges from 0 to 24 . In addition, it is established as a cut-off score of "14" points, which means that individuals having a higher score than 14 show disability. ${ }^{13}$

\section{Measurement}

The GraphPad Prism was used for statistical analysis and a significance level of $5 \%(p<0.05)$ was considered for all statistical tests. In order to analyze the relationship between psychological aspects with low back pain, the sample was stratified into two categories: adults and elderly group. Chi Square and Fisher's Exact Test were used to evaluate the association between stress and LBP. The Mann-Whitney's test was used to compare the groups considering psychological aspects. In order to investigate if functional disability has an association with LBP, we used the Spearman's Correlation for the outcomes depression and anxiety and Fisher's Exact Test for the outcome anxiety. The effect size was also calculated in order to check the difference between the groups.

\section{Results}

Eighty-four individuals (41 adults and 43 elderly) were enrolled at this study. No statistically significant differences were observed between the groups for both adults and elderly except for the pain pressure threshold which was smaller in individuals with LBP. There was a predominance of women, especially in the low back pain groups. The sample characterization and socio-demographic characteristics are shown in Table 1. When analyzing the relationship between psychological aspects (stress) and low back pain (LBP) and functional disability, there was a statistically significant association between older adults with low back pain and stress (Fisher's exact test, $p=0.001$, Phi $=0.522$ ), with a moderate effect size (Cohen, 1988). However, among adults this association was not observed $(p=0.209)$. Elderly individuals with LBP showed worse functional status when compared to control group (Mann-Whitney=29.50; $p<0.001, \mathrm{~d}=2.02$ ), supported by the strong effect size. Similar results were observed concerning depression (Mann-Whitney=132.0, $p=0.015, \mathrm{~d}=0.33$ ) 
and anxiety (Mann-Whitney=125.0, $p=0.007, \mathrm{~d}=0.41$ ). In adults, are shown in Figure 1. In individuals with LBP, it was also observed significant differences were found in anxiety (Mann-Whitney $=124.5$, a relation between functional disability with depression ( $\mathrm{rs}=0.61$; $p=0.025, \mathrm{~d}=0.70)$ and disability (Mann-Whitney=112.5, $p=0.008, \quad p<0.001)$ and anxiety $(\mathrm{rs}=0.55, p=0.002)$, being these data shown in $\mathrm{d}=0.80$ ). On the other hand, no differences were observed regarding figure 2. Additionally, no association between stress and functional depression status (Mann-Whitney=204.5, $p=0.885$ ). All these data disability was observed (Fisher's exact test $=0.440 ; p=0.602$ ).

Table I Sample characteristics

\begin{tabular}{|c|c|c|c|c|c|c|c|}
\hline & & \multicolumn{3}{|l|}{ Adults } & \multicolumn{3}{|l|}{ Elderly } \\
\hline & & $\begin{array}{l}\text { Control } \\
\text { group }\end{array}$ & $\begin{array}{l}\text { Low back pain } \\
\text { group }\end{array}$ & $\mathbf{P}$ & $\begin{array}{l}\text { Control } \\
\text { group }\end{array}$ & $\begin{array}{l}\text { Low back pain } \\
\text { group }\end{array}$ & $\mathbf{p}$ \\
\hline \multirow[t]{2}{*}{$N$} & & 21 & 20 & & 23 & 20 & \\
\hline & Mean & 31.38 & 30.75 & 0.84 & 71.69 & 68.85 & \multirow[b]{2}{*}{0.08} \\
\hline \multirow[t]{2}{*}{ Age } & $\begin{array}{l}\text { Standard } \\
\text { Deviation }\end{array}$ & 1.85 & 2.42 & & 1.09 & 1.15 & \\
\hline & Mean & 21.01 & 22.81 & 0.57 & 22.36 & 26.81 & \multirow[b]{2}{*}{0.18} \\
\hline BMI & $\begin{array}{l}\text { Standard } \\
\text { Deviation }\end{array}$ & 2.41 & 1.93 & & 2.39 & 2.1 & \\
\hline \multirow[b]{2}{*}{$\begin{array}{l}\text { Pain Pressure } \\
\text { Threshold }\end{array}$} & Mean & 7.3 & 5.31 & $0.005^{*}$ & 7.47 & 6.04 & \multirow[b]{2}{*}{$0.002^{*}$} \\
\hline & $\begin{array}{l}\text { Standard } \\
\text { Deviation }\end{array}$ & 0.45 & 0.49 & & 0.31 & 0.28 & \\
\hline \multirow{2}{*}{ Gender } & Male & 05 (23.8\%) & 06 (30.0\%) & 0.73 & 04 (17.4\%) & OI (5.0\%) & \multirow{2}{*}{0.35} \\
\hline & Female & $16(76.2 \%)$ & 14 (70.0\%) & & 19 (82.6\%) & 19 (95.0\%) & \\
\hline
\end{tabular}

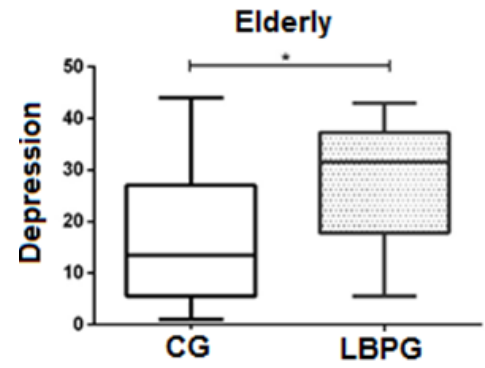

Adults

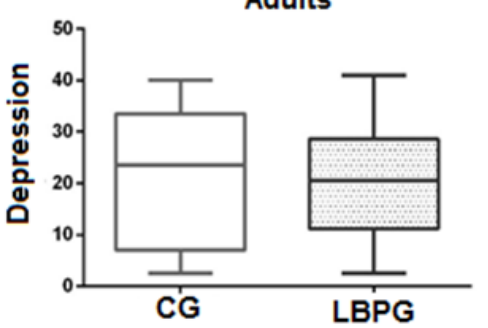

Elderly
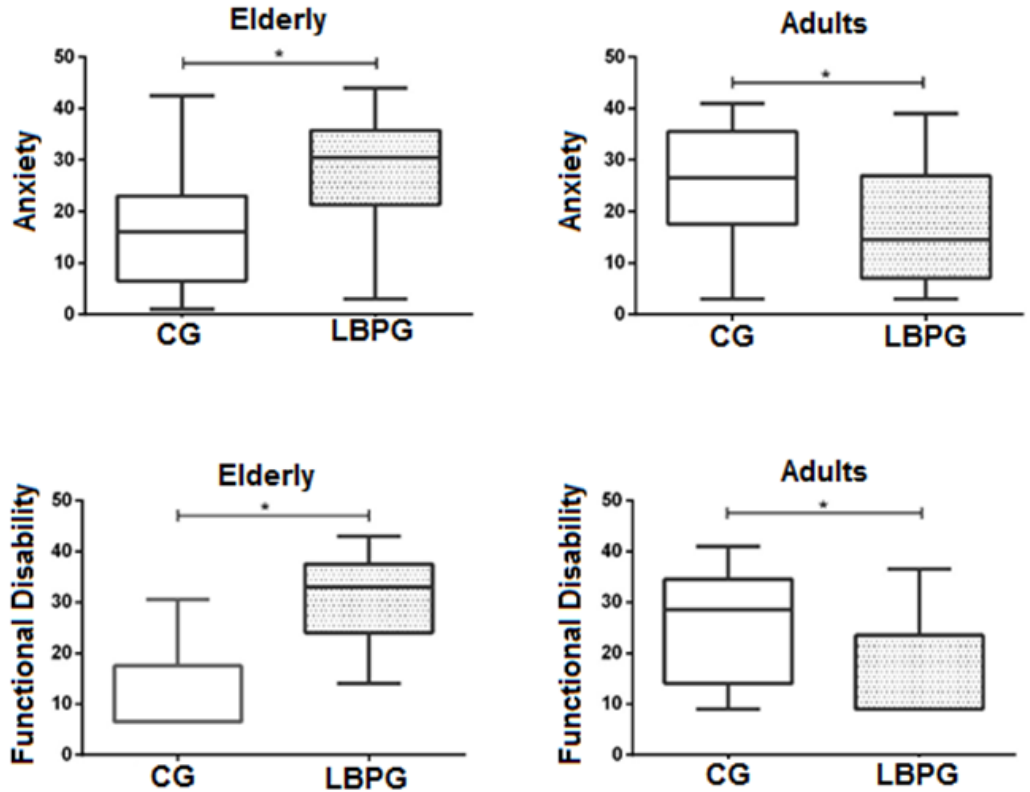

Figure I Comparison between Control Group (CG) and Low Back Pain Group (LBPG) regarding depression. Anxiety and functional disability (*Statistically significant, Mann-Whitney Test, $p<0.05)$. 

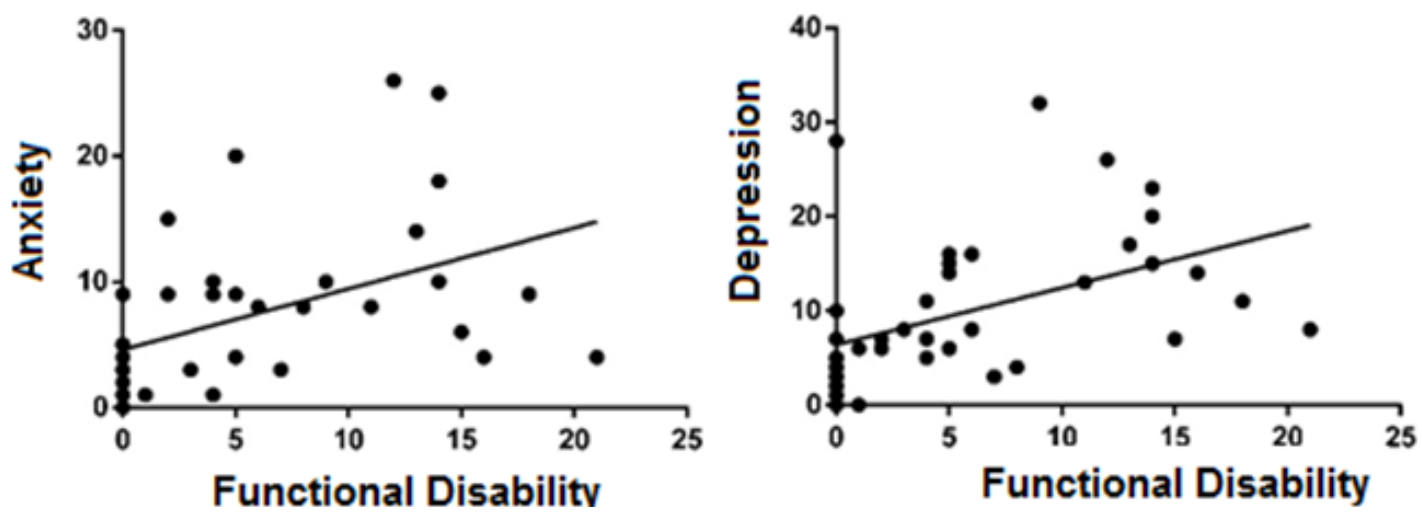

Figure 2 Correlation between Functional Disability and Anxiety and Depression in individuals with Low Back Pain.

\section{Discussion}

In this study, no differences were observed between the anthropometric and demographic characteristics of the groups and, therefore, these variables were not considered confounding factors. In women's groups, low back pain predominated as in other studies. ${ }^{4,14}$ This finding may be related to the fact that women have lower resistance to weight, fragile joints, shorter stature, lower bone mass and a higher percentage of body fat, making them more susceptible to the occurrence of low back pain. ${ }^{15,16}$ There was an association between the elderly with low back pain and stress. The onset of the low back pain process may characterize the persistence of stressors, whether in frequency or intensity. ${ }^{11}$ Pain inducing features similar to the stress process, through activation of the pituitary gland that secretes adrenocorticotropic hormone by acting on the adrenal cortex to secrete cortisol. ${ }^{17}$

The fact that this association occurs among the elderly may be due to the importance of previous experience with a major factor in the emergence of such conditions, since there is often the combination of the restrictions on activities of daily living be due to the painful process. ${ }^{18}$ A study conducted with women in Spain indicated that DLC was more frequent among the 45-64 age group and $\geq 65$ years than among the groups age group (16-24 years). ${ }^{19}$ Prior experiences pain produce reactions that cause stress and anxiety generating as a consequence a reduction in pain threshold, feedback facilitating this process. ${ }^{20}$

It was also noticed a statistically association between elderly with chronic LBP and functional disability, showing that seniors who have low back pain show up with greater functional disability. These data is in agreement with other studies who have pointed out how chronic pain may affect job, social and family activities. ${ }^{4,20}$ Regarding social aspects, individuals usually remain in their home environment, increasing their chance of developing anxiety and depression, the most common conditions observed in these individuals. ${ }^{22}$ In addition, there is evidence that anxiety may be a predisposing factor. ${ }^{23-25}$ Taking into account individuals with low back pain, they may be anxious about their symptoms and future, especially in relation to physical limitation. ${ }^{26}$ At this study, a correlation between depression and LBP was also described, more frequently among the elderly, such as in other studies. ${ }^{2,4,14,18,24,27}$ Considering that depression has been associated with high levels of pain and small pressure pain threshold, one may assume that depression may develop after the LBP and not otherwise. ${ }^{28-30}$ Researchers suggest pharmacological and nonpharmacological approaches for clinical treatment in patients with chronic suffering. ${ }^{31}$ Other treatments can be performed because a study that developed a program of McGill exercises and exercise at Sahrmann level in women with low back pain showed that both intervention groups were effective in reducing pain. ${ }^{32}$

Individuals with higher levels of depression tend to become isolated and less motivated, and may reduce self-efficacy and increase the chances of depressive symptoms as well as fear and depression..$^{33}$ For this, researchers indicate multidisciplinary treatments, ${ }^{34-36}$ since that depressive episodes often begin after the onset of the pain problem due to the limitations of the disease.${ }^{37}$ Moreover, a prospective study showed that patients with depression are 2.3 times more likely to develop back pain compared to those who did not report depression. However, it is worth mentioning that not all individuals with chronic pain are depressed, due to the different effects that pain causes in people's lives, as well as to the ability of each individual to exert some control over pain and their life. ${ }^{38}$ Based on the observed results, a relationship between functional disability, anxiety, depression and stress in elderly patients with chronic low back pain may be suggested. In addition, it indicates the existence of a relationship between depression and anxiety in functional disability, regardless of age. Therefore, we may propose that chronic LBP is related to psychological aspects and functional disability in the elderly, being important factors at the onset, severity, exacerbation or maintenance of this type of chronic pain. Based on the observed results, a relationship between functional disability, anxiety, depression and stress in elderly patients with chronic low back pain may be suggested. ${ }^{39}$ In addition, it indicates the existence of a relationship between depression and anxiety in functional disability, regardless of age. Therefore, we may propose that chronic LBP is related to psychological aspects and functional disability in the elderly, being important factors at the onset, severity, exacerbation or maintenance of this type of chronic pain. Study limitations although the scales used in the present study are validated, they are self-reported by the research participant and may present bias in the response.

\section{Acknowledgments}

None.

\section{Conflicts of interest}

There is no potential conflict of interest for this article.

\section{Funding}

None.

\section{References}

1. Almeida OP. Mini mental status exam and the diagnosis of dementia in Brazil. Arq Neuro-Psiquiatr. 1998;56(3). 
2. Altuğ F, Kavlak E, Kurtca MP, et al. Comparison of pain intensity, emotional status and disability level in patients with chronic neck and low backpain. J Back Musculoskelet Rehabil. 2015;28(3):505-508.

3. Asmundson GJG. Anxiety sensitivity and chronic pain: Empirical findings, clinical implications, and future directions. In Taylor S, editors. Anxiety sensitivity: Theory, research, and treatment of the fear of anxiety Mahwah, NJ: Erlbaum; 1999:269-286.

4. Asmundson GJG, Wright KD, Hadjistavropoulos HD. Anxiety sensitivity and disabling chronic health conditions: State of the art and future directions. Scandinavian Journal of Behaviour Therapy. 2000:05.

5. Beck AT, Epstein N, Brown G, et al. An inventory for measuring clinical anxiety: Psychometric properties. J Consult Clin Psychol. 1988;56(6):893-897.

6. Bener A, Verjee M, Dafeeah EE, et al. Psychological factors: anxiety, depression, and somatization symptoms in low back pain patients. $J$ Pain Res. 2013:(4).

7. Boersma K, Linton SJ. Screening to identify patients at risk: Profiles of psychological risk factors for early intervention. Clin J Pain. 2005;21:38 43.

8. Brown GK. A causal analysis of chronic pain and depression. J Abnorm Psychol. 1990;99(2):127-137.

9. Canaway A, Pincus T, Underwood M, et al. Is an enhanced behaviour change intervention cost-effective compared with physiotherapy for patients with chronic low back pain? Results from a multicentre trial in Israel. BMJ Open. 2018. 8(4):e019928.

10. Cherkin DC, Sherman KJ, Balderson BH, et al. Effect of mindfulnessbased stress reduction vs cognitive behavioral therapy or usual care on back pain and functional limitations in adults with chronic low back pain: a randomized clinical trial. JAMA. 2016: 315(12):1240-1249.

11. Cunha JA. Manual da versão em português das Escalas Beck. São Paulo: Casa do Psicólogo. 2011.

12. Cohen J. Statistical power analysis for the behavioral sciences. Hillsdale, New Jersey: Lawrence Erbaum, 2nd ed. 1988.

13. Dae-Hyun Kim, Tae-Ho Kim. Comparison of the effects of stability exercise and balance exercise on muscle activity in female patients with chronic low back pain. J Exerc Rehabil. 2018;14(6):1053-1058.

14. Dersh J, Gatcher RJ, Mauer TG, et al. Prevalence of psychiatric disorders in patients with chronic disabling occupational spinal disorders. Spine. 2006;31(10):1156-1162.

15. Ellegaard H, Pedersen Bd. Stress is dominant in patients with depression and chronic low back pain: A qualitative study of psychotherapeutic interventions for patients with non-specific low back pain of 3-12 months duration. BMC Musculoskeletal Disorders. 2012; 6;13:166.

16. Fehrmann E, Kotulla S, Fischer L, et al. The impact of age and gender on the ICF-based assessment of chronic low back pain. Disabil Rehabil. 2018:12.

17. Figueiredo VF, Pereira LSM, Ferreira PH, et al. Functional disability, depressive symptoms and low back pain in elderly. Fisioter Mov 2013;26(3):549-557.

18. Foster NE, Thomas E, Bishop A, et al. Distinctiveness of psychological obstacles to recovery in low back pain patients in primary care. Pain. 2010;148(3):398-406.

19. Froud R, Patterson S, Eldridge S, et al. A systematic review and and meta-synthesis of the impact of low back pain on people's lives. BMC Musculoskelet Disord. 2014;21:15:50.

20. Gatchel RJ. Clinical essentials of pain management. Washington, DC: American psychological association. 2005.
21. Garcia JBS, Hernandez-Castro JJ, Nunez RG, et al. Prevalence of low back pain in Latin America: a systematic literature review. Pain Physician. 2014;17(5):379-391

22. Gorenstein C,Andrade LHSG. Inventário de depressão de Beck: propriedades psicométricas da versão em português. Rev Psiq Clin. 1998;25(5):245-250

23. Groot KI, Boeke S, Van den Berge HJ, et al. Assessing short and long-term recovery from lumbar surgery with pre-operative biographical, medical and psychological variables. British Journal of Health Psychology. 1997.

24. Guclu DG, Guclu O, Ozaner A, et al. The relationship between disability, quality of life and fear-avoidance beliefs in patients with chronic low back pain. Turk Neurosurg. 2012;22(6):724-31.

25. Jiménez-Sáncheza S, Fernández-de-las-Penas C, Carrasco-Garridoa P, et al. Prevalence of chronic head, neck and low back pain and associated factors in women residing in the Autonomous Region of Madrid (Spain). Gac Sanit. 2012;26(6):534-40.

26. Kamper SJ, Apeldoorn AT, Chiarotto A, et al. Multidisciplinary biopsychosocial rehabilitation for chronic low back pain: Cochrane systematic review and meta-analysis. BMJ. 2015;350:444

27. Karp JF, Gao X, Wahed AS, et al. Effect of Problem-Solving Therapy Versus Supportive Management in Older Adults with Low Back Pain and Depression While on Antidepressant Pharmacotherapy. Am J Geriatr Psychiatry. 2018;26(7):765-777.

28. Karp JF, Weiner DK, Dew MA, et al. Duloxetine and care management treatment of older adults with comorbid major depressive disorder and chronic low back pain: results of an open-label piloty study. Int J Geriatr Psychiatry. 2010; 25(6):633-42.

29. Koo TK, Guo J, Brown CM. Test-Retest Reliability, repeatability, and sensitivity of na automated deformation-controlled indentation on pressure pain threshold measurement. J Manipulative Physiol Ther. 2013 ;36(2):84-90.

30. Kregel J, Meeus M, Malfliet A, et al. Structural and functional brain abnormalities in chronic low back pain: a systematic review. Semin Arthritis Rheum. 2015;45(2):229-237.

31. Lipp MEN. Estresse emocional: a contribuição de estressores internos externos. Rev Psiq Clin. 2001;28(6):347-349.

32. Meucci RD, Fassa AG, Faria NMX. Prevalence of chronic low back pain systematic review. Rev Saúde Pública. 2015. p.49.

33. Meyer K, Tschopp A, Sprott H, et al. Association between catastrophizing and self-rated pain and disability in patients with chronic low back pain. $J$ Rehabil Med. 2009;41(8):620-5.

34. Mclean SA, Williams DA, Harris RE, et al. Momentary relationship between cortisol secretion and symptoms in patients with fibromyalgia. Arthritis Rheum. 2005;52(11).

35. McNeill C. Management of temporomandibular disorders: concepts and controversies. J Prosthet Dent. 1997;77(5):510-522.

36. Nusbaum L, Natour, Ferraz MB, et al. Translation, adaptation and validation of Rolland-Morris questionnaire - Brazil Roland-Morris. Braz J Med Biol Res. 2001;34(2):203-10.

37. Preuper HR, Reneman MF, Boonstra AM, et al. Relationship between psychological factors and performance-based and self-reported disability in chronic low back pain. Eur Spine J. 2008;17(11):1448-56.

38. Rudy TE, Weiner DK, Lieber SJ, et al. The impact of chronic low back pain on older adults: a comparative study of patients and controls. Pain 2007;131(3):293-301.

39. Von Elm E, Altman DG, Egger M, et al. The Strengthening the Reporting of Observational Studies in Epidemiology (STROBE) statement: guidelines for reporting observational studies. Ann Intern Med. 2007;61(4):344-9. 